\title{
Dentin Hypersensitivity-Etiology, Treatment Possibilities and Other Related Factors: A Literature Review
}

\author{
Alessandra Bühler Borges, Daphne Câmara Barcellos, Carlos Rocha Gomes Torres, Alexandre Luiz Souto Borges \\ Ana Lucia Marsilio, Cláudio Antonio Talge Carvalho
}

\begin{abstract}
Dentinal hypersensitivity is a common painful condition of the teeth, associated with the exposure of the dentin to external environment of the mouth. However, little is known about the etiology and predisposing factors, which can compromise management. The aim of this paper is to summarize existing information about epidemiology, mechanisms, etiology, differential diagnosis and treatment.
\end{abstract}

Clinical relevance: A better understanding of the etiology and factors involved in dentin hypersensitivity will help the management of the hypersensitivity teeth.

Keywords: Dentinal hypersensitivity, Dentin sensitivity/etiology, Dentin sensitivity/therapy.

How to cite this article: Borges AB, Barcellos DC, Torres CRG Borges ALS, Marsilio AL, Carvalho CAT. Dentin Hypersensitivity-Etiology, Treatment Possibilities and Other Related Factors: A Literature Review. World J Dent 2012;3(1):60-67.

Source of support: Nil

Conflict of interest: None declared

\section{INTRODUCTION}

\section{Definition}

Dentinal hypersensitivity is defined as an exaggerated response to a stimulus that usually causes no response in a healthy tooth, ${ }^{1,2}$ and also, an exaggerated response to a nonharmful stimulus. The nonharmful stimuli are the thermal, tactile or osmotic stimuli that when applied on the exposed dentin, evoke pain without causing pathologic alterations to the dentin-pulpar complex. ${ }^{3}$

\section{Epidemiology}

About 8 to $30 \%$ of the adult population present dentinal hypersensitivity and the most affected age is among 20 and 30 years old. ${ }^{4}$ The first premolars are the most affected teeth, reaching more than half of the cases and the most affected region is the cervical area of the buccal surface. ${ }^{5}$

\section{Dentine Hypersensitivity Mechanisms}

The intradentinal nerves are confined to the predentin and the most pulpal part of dentin. This supports the likelihood of an indirect stimulatory mechanism, evoking pain. ${ }^{5-7}$

Several theories try to explain the dentinal hypersensitivity although none of them leads to a perfect understanding of how the stimuli applied in this surface can cause pain.
- Transduction theory: According to this theory, the odontoblast has a special sensory function and the functional complex with the nerve ending in or near the odontoblastic layer acts as an excitatory synapse. The odontoblast and its process have been perceived as a transducer mechanism. ${ }^{6}$

- Modulation theory: According to this theory the nerve impulses in the pulp are modulated through the liberation of polypetides from the odontoblasts, when injured. These substances may selectively alter the permeability of the odontoblastic cell membrane through hyperpolarization, so that pulp neurons are more prone to discharge upon receipt of subsequent stimuli. ${ }^{6}$

- Hydrodynamic theory: According to Brännström's hydrodynamic theory, ${ }^{8}$ when an appropriate stimulus is applied to the outer dentin surface, there is a displacement of the contents of the dentinal tubules, that gives rise to a mechanical stimulation of the pain at the pulpodentinal border. This theory is the most widely accepted until now and consider that the stimulation of the nerve endings next to the odontoblastic layer is provoked by the variation of the intrapulpal pressure toward the pulp or in the opposite direction, depending on the stimulus nature. The nerve fibers stimulation occurs because of the deformation of these fibers, caused by the fluid movement, leading to a widening of the nerve membrane ionic canals, allowing the entrance of $\mathrm{Na}^{++}$in the cell, depolarizing the fibers and provoking pain. ${ }^{3}$

\section{Dentinal Tubules Morphology}

The number of tubules per area next to the pulpal surface in comparison to the outer areas is of $4: 1$. So, the superficial dentin is less permeable than the deep dentin. ${ }^{9}$

The dentinal tubules diameter decreases progressively toward the pulp to the enamel in the coronary dentin. ${ }^{10}$ The diameter of the tubules in the deep dentin region is 3 to 4 $\mathrm{mm}$ and in peripheral dentin is $1 \mathrm{~mm} .{ }^{11}$

Microscopically, it has been shown that hypersensitive dentinal areas present wider tubules and in increased number than nonsensitive areas. This provides evidence that stimulus transmission across dentin in hypersensitive teeth is mediated by hydrodynamic mechanism. ${ }^{12}$ 
When this theory is accepted as the explanation of the stimulus transmission across dentin, it must be assumed that dentinal tubules are open at the exposed dentin surface and patent through the pulp. This would help explain why some patients with exposed cervical dentin exhibit dentinal hypersensitivity and others do not. The open tubules are not presented over the whole surface of dentin and are irregularly distributed. This can be observed by probing the area where only the open tubules areas respond with pain. ${ }^{12}$

Dentinal hypersensitivity is most likely to occur in younger people who experience rapid exposure of the root surface. ${ }^{6}$ Old people, in spite of presenting root exposition most frequently, usually do not show painful sensitivity; this can be explained by the following factors:

- Mineral deposition inside the tubules (dentinal sclerosis)

- Reduction in the number of tubules

- Pulp chamber reduction due to an increase of reparative dentin

- A reduction in cellularity, vascularity and nerve fibers in the pulp.

Pulp pressure is greater than the atmospheric pressure, this explains why the dentinal fluid is constantly flowing through the permeable exposed dentin, although in very low rates to activate the pulpal mechanoreceptors. Any event that increases the pressure gradient, increasing the fluid movement, may evoke pain. ${ }^{13}$ The dentinal hypersensitivity triggering off may be a thermal origin, evaporative, tactile and osmotic: ${ }^{14}$

- Thermal: The pain is caused by the temperature variation which causes fluid contraction, when cold, or expansion, when hot, leading consequently to its movement;

- Evaporative: Through the application of a brief air blast on the tooth, leading to a evaporation of the dentinal canals fluid which are open;

- Tactile: The simplest device for testing hypersensitivity is scrubbing a sharp probe along the cementum-enamel junction. This provokes the fluid movement probably due to a vibration mechanism;

- Osmotic: The pain is provoked in the presence of sweet substances. Because they are hypotonic, they cause an osmotic pressure in dentin, which leads to a movement of the dentinal fluid toward the sweet substance.

\section{Etiology}

An understanding of the etiology of any disease or condition is essential for effective prevention and treatment. The current knowledge of the etiology of dentinal hypersensitivity is still limited and the factors that lead to a dentinal exposure are still uncertain. ${ }^{15}$

Dentin in normal conditions is coated by enamel and cementum, not presenting sensitivity to external stimuli.
Thus, the dentin starts presenting hypersensitivity only when exposed to the mouth environment, after the wear of the protection structures.

The enamel layer can be removed by attrition from occlusal wear and parafunctional habits, toothbrushing abrasion, erosion from acids, coronal fracture, abfractions or defective fillings. Gingival recession, periodontal disease, periodontal surgery and incorrect toothbrushing can expose root surfaces. Once exposed, the thin cementum layer (20$25 \mathrm{~mm}$ ) is easily removed by periodontal root scaling, abrasive pastes, acidic foods and toothbrushing. Besides these factors, about $10 \%$ of individuals, the enamel and cementum do not meet exposing a dentin area. ${ }^{16}$

Dentinal hypersensitivity may be closely related to the occlusal stress, where the occlusal forces would lead to an increase of the intrapulpal pressure, provoking an increase of the movement speed of dentinal fluid, unleashing the hypersensitivity. ${ }^{3}$

Dentinal hypersensitivity has chronic features showing exacerbation periods. One must consider, thus, the psychological component of the chronic pain, because the psychic tension may reduce the tolerance threshold to external stimuli. ${ }^{16}$ It is known that the pain mechanism is complex and that the neural activity initiated by a peripheral stimuli may be modified by physic and psychological factors. ${ }^{17}$

There are no evidences concerning to the presence or not of pulpal changes associated with dentinal hypersensitivity. ${ }^{6,7}$ Some authors believe that there may be changes in the pulpodentin complex as a consequence of the exposed dentine and penetration of solutes and toxines from the outer surface, especially if there is a cavity or caries, or if plaque is on dentin. ${ }^{10,18}$ But, according to Addy and Pearce ${ }^{15}$ (1994), the phenomenon of bacterial-associated sensitivity has a defined pathology and therefore is not dentinal hypersensitivity.

\section{Differential Diagnosis}

It is important that the collection of a correct dentinal hypersensitivity diagnosis, differentiating it from other pathologies which present similar symptoms, allowing the establishment of an adequate treatment program. A careful diagnosis includes a history and a thorough clinical and radiographic examination. The etiological and predisponent factors investigation depend on a thoughtful anamnese and also on detailed clinical and radiographical inspections.

It is necessary that the information intake about the tooth cleaning habits, including the frequency and duration, type of toothbrush, brushing technique, interval of toothbrush change and pressure applied during the toothbrushing.

It is also important to investigate about the habits that may lead to teeth or gums trauma. The contact with erosive 
agents must be questioned including those arising from living environments, working, medications, illness and, most commonly, diet. ${ }^{19}$

In general, the related history from the patient agrees with the following aspects: Pain provoked by thermal stimuli (cold and/or hot food, air during the talking or even aspiration), chemicals (sweet and acid food), tactile (brushing and probing); localized pain with great definition of dental element; acute pain with short duration, which vanishes with stimulus removal; spontaneous remission periods, most of the times coincident with the patient anxiety state. $^{3}$

Although at times pulpal inflammation complicates the symptomatology tooth, hypersensitivity differs from pain arising in the pulp due to inflammation. When a stimulus is applied to a hypersensitive tooth, the patient can readily locate the source of pain and discomfort, while the pulpal pain may be lasting, intermittent and throbbing. The simplest method for the differentiation is the application of an air blast on the tooth, because the air stimulation usually reflects dentinal hypersensitivity rather than a problem of pulpal origin. ${ }^{1}$ When performing the dentinal hypersensitivity diagnosis, other pathologies and dental defects must be considered including the cracked tooth syndrome, fractured restorations, chipped teeth, caries, postrestorative sensitivity and teeth in hyperfunction, by examining carefully the affected region in order to exclude the alternative causes of pain. ${ }^{1}$

\section{Dentinal Hypersensitivity Treatment}

There are different kinds of dentinal hypersensitivity treatment available, nevertheless, the ideal desensitizer agent, according to Grossman ${ }^{20}$ (1935), should be nonirritating to the pulp, be relatively painless, be easily applied, act quickly, be permanently effective and not discolor tooth structure.

There are two basic methods to obtain the results during dentine hypersensitivity evaluation procedure: To use either a constant level of stimulus intensity and have the patient on categorical or interval scale; or to increase gradually the stimulus intensity and record the patient's first response. ${ }^{21}$ Although the second method is the most indicated one, it is usually subjective and depends on the patient response to the stimuli and within these, there are those which promote more pain causing the patient to be more apprehensive and tense waiting for the moment of pain. Due to this fact, for several times, the patients do not inform correctly the moment of the response to the applied stimulus, which can alter the evaluation results.

\section{Desensitizing Agents Utilized in the Office}

\section{Calcium Hydroxide}

The treatment consists of the application of a calcium hydroxide paste for 3 to 5 minutes through the burnishing with a wooden stick or rubber tip. The real calcium hydroxide action mechanism is unknown, but it is suggested that it may block dentinal tubules or promote peritubular dentin formation. It is believed that the high $\mathrm{pH}$ provokes the odontoblastic process protein coagulation, leading to a closure of the tubules through precipitation of these proteins, decreasing the hydraulic conductance. ${ }^{22}$

\section{Iontophoresis}

Iontophoresis is the process of introducing ionic drugs into body surfaces for therapeutic purposes, and is highly suited to therapy of conditions at or near the body surface. It requires that: A charged drug be delivered at the electrode of the same polarity, the condition or disease under treatment be at or near the surface and, a source of direct current with appropriate means of application, be used..$^{23,24}$

\section{Fluoride Compounds}

When in contact with the mineralized tooth structures, fluoride ions are claimed to cause a reduction in diameter of the dentinal tubules by precipitating $\mathrm{CaF}_{2}$ crystals. ${ }^{16}$ The speculated mode of action is the formation of a calcified barrier blocking the tubule openings. Since calcium fluoride is an unstable compound, it provides a short period of desensitizing effect. This indicates the necessity of several applications to reduce the pain. Among the most used fluoride compounds are the 5\% sodium fluoride varnish (Duraphat, Colgate-Palmolive Company), 2\% neutral sodium fluoride and $1.23 \%$ acidulated fluoride phosphate gel. ${ }^{21}$ Fluoride compounds, such as amine fluoride, ${ }^{25}$ stannous fluoride ${ }^{26}$ and titanium tetrafluoride ${ }^{27,28}$ have also been employed in order to reduce dentinal sensitivity.

\section{Fluoride Iontophoresis}

Fluoride iontophoresis is applied to allow $\mathrm{F}^{-}$ions penetration more deeply in the dentinal tubules. The exact mechanism by which fluoride iontophoresis produces desensitization of dentine is not known. The desensitization could occur by two mechanisms: Intratubular microprecipitation of $\mathrm{CaF}_{2}$ affecting dentin permeability and an effect of the fluoride on the neural transduction mechanism..$^{23,29}$

It consists of a $2 \%$ sodium fluoride application with the help of an electrode. When $\mathrm{NaF}$ dissolves into solution, its ions become negatively charged. This is placed on the tooth with a cotton tipped rod which is the negative electrode. An electric current is then passed through the tooth to the 
other electrode which is held by the patient completing the circuit. Thus, the fluoride ions are pushed into the dentinal tubules. $^{22}$

\section{Strontium Chloride}

The exact mechanism of strontium chloride is unclear. Strontium ions penetrate dentin deeply and replace calcium, resulting in recrystallization in the form of a strontium apatite complex. ${ }^{16,22}$ Some strontium chloride-based formulations are found in the market as varnishes or dentifrices. $\operatorname{Ross}^{30}$ (1961) and Kishore et a ${ }^{31}$ (2002) treated patients with $10 \%$ strontium chloride and observed that these desensitizing agents significantly reduced dentin hypersensitivity. However, Zappa ${ }^{21}$ (1994) do not support the effectiveness of $10 \%$ strontium chloride hexahydrate toothpaste in relieving the pain of tooth hypersensitivity and in comparison with other agents, the efficacy of strontium chloride is uncertain. Examples of strontium chloride-based desensitizing agents are the Zarosen varnish (Cetylite Ind. Inc., USA) and the Sensodyne toothpaste for sensitive teeth (Block Drug Corp, USA).

\section{Oxalate Desensitizers}

Oxalate ions react with calcium to form insoluble calcium oxalate crystals that occlude the dentinal tubules, decreasing dentin permeability. ${ }^{16}$ Although oxalates appear to be one of the best topical treatment for blocking tubules, they have the same drawback as all topic treatments, as the eventual dissolution of the surface precipitate that forms the barrier by saliva. ${ }^{23}$ Example of oxalate-based desensitizing agents is Super Seal (Phoenix Dental, Inc., Fenton, MI, USA) and Bisblock (Bisco, Schaumburg, IL, USA).

\section{Potassium Nitrate}

A potential desensitizing agent like potassium nitrate acts in two ways: Decreasing fluid flow through the tubules by occluding (clogging) them; decreasing the level of activity of the dental sensory nerves, thus preventing the pain signals to be transmitted to the central nervous system. Potassium ion of potassium nitrate diffuses through the dentinal tubule and reaches the pulp-sensory complex and forms a region of greatly increased concentration (with $\mathrm{K}^{+}$ions) which subsequently depolarizes the pulpal sensory complex and reduces pain transmission. ${ }^{32}$ Examples of potassium nitrate based desensitizing agents are UltraEZ (Ultradent Products, Inc) and Desensibilize (FGM, Brazil).

\section{Application of a Waterproof Film and Restorative Procedures}

These desensitizing agents have a blocking action mechanism through the sealing of exposed dentinal tubules, decreasing dentin permeability and hydraulic conductance.
- Varnishes: Varnishes promote a waterproof film formation. The most common are the copal varnishes and varnishes with desensitizing agents like strontium chloride and sodium fluoride.

- Adhesive systems: The adhesive systems are indicated for dentine hypersensitivity treatment, since they have hydrophilic primers, allowing an efficient dentin bonding. The use of HEMA as a hydrophilic primer is common in many modern dentin bonding systems. The Gluma Desensitizer (Heraeus Kulzer, GMBH) is an aqueous solution of 35\% HEMA and 5\% glutaraldehyde. Glutaraldehyde is an effective fixative of flocculating agent and have the capacity to form a coagulation 'plug' within the dentinal tubules. ${ }^{33}$ Some products replace the glutaraldehyde with benzalkonium chloride and include a small concentration of fluoride in the formulation. Benzalkonium chloride is a desinfectant. The main benefit of the nonglutaraldehyde agents is that they are kinder to the tissue. Glutaraldehyde eventually causes a transient tissue burn and allergies and must be carefully used. ${ }^{33}$ The new trend of treatment of the dentinal hypersensitivity is the use of the self-etching adhesive systems. According to Türkün ${ }^{34}$ (2003), treating the enamel and dentin with self-etching adhesive systems instead of employing a conventional total-etch procedure prevent the collapse of collagen network. Additionally, the self-etching primers need no etching, rinsing and drying, so that the risk of over-etching and over-drying of the dentin is eliminated. They are simple to apply in clinical situations, and reduce the technique sensitivity of the bonding procedure. The self-etch adhesive systems Futurabond M (Voco, GMBH), Adper SE Plus (3M ESPE, USA) and One-Up Bond F plus (Tokuyama, Japan) are examples of self-etching systems that use HEMA as a hydrophilic primer.

- Restorative procedures: These procedures commonly use composite resins and glass ionomer cements. They are usually applied when esthetic and functional reestablishment of deeper lesions are necessary, besides the dentinal hypersensitivity control.

\section{Laser Light}

- Nd:YAG laser: Previous scanning electron microscopic studies showed that Nd:YAG laser could cause melting of dentin and closure of exposed dentinal tubules without dentin surface cracking ${ }^{35}$ resulting in a reduction of permeability and hydraulic conductance. ${ }^{23}$ The sealing depth of Nd:YAG on human dentinal tubules is about $4 \mathrm{~mm}$ in the center and $3 \mu \mathrm{m}$ at the margin of the lased surfaces. ${ }^{35}$ Hsu et $\mathrm{al}^{36}$ (2006) evaluate the combined occluding effects of fluoride-containing dentin 
desensitizer and Nd:YAG laser irradiation on human dentinal tubules, and observed that the occluding agent was thus 'burned into' the dentinal tubules, reducing the hypersensitivity and increasing the duration of the desensitizing effect. Kumar and Mehta $^{37}$ (2005) observed that Nd:YAG laser, when used alone, is effective in the treatment of dentin hypersensitivity, and the combination of the Nd:YAG laser and $5 \% \mathrm{NaF}$ varnish seems to show an impressive efficacy, when compared to either treatment alone.

- Er:YAG: According to Birang et $\mathrm{al}^{38}$ (2007), the use of Er:YAG laser, with the parameters $3 \mathrm{~Hz}$ and $100 \mathrm{~mJ}$, was effetive to decrease the pain of dentinal hypersensitivity. However, there are a lot of ambiguous points in the mechanisms of Er:YAG as this laser is absorbed by water molecules in hydroxyapatites, can cause ablation of dentin surface and is opposite to the sealing of dentinal tubules. Schwarz et $\mathrm{al}^{39}$ (2002) stated that an Er:YAG laser with parameters of the $80 \mathrm{~mJ} /$ pulse and $3 \mathrm{~Hz}$ was effective to desensitizing hypersensitive dentine.

- $\mathrm{CO}_{2}$ : Zhang et al ${ }^{40}$ (1998) observed that the $\mathrm{CO}_{2}$ laser reduced cervical dentinal hypersensitivity by $50 \%$, without any thermal damage to the pulp. However, scanning electron microscopy showed that the effect of laser energy on dentin varied from charring, cratering, pore and/or fissure formation, fracturing and cracking, up to melting, and that the dentinal tubules were not sealed. Slutzky-Goldberg et $\mathrm{al}^{41}$ (2008) tested $\mathrm{CO}_{2}$ laser energy as a possible alternative treatment for prevention of dentin hypersensitivity in restorative dentistry. They stated that the $\mathrm{CO}_{2}$ laser energy appears to be a promising tool for use in the clinical setting.

- Low-level laser therapy: This relies on laser-induced changes to neural transmission networks within the dental pulp, rather than alterations to the exposed dentine surface, as in other treatment modalities. The most used in dentinal hypersensitivity treatments are the GaAlAs and HeNe lasers. ${ }^{42}$ According to Tengrungsun and Sangkla ${ }^{43}$ (2008), GaAlAs laser treatment seems to be beneficial and can be an alternative treatment for dentin hypersensitivity.

\section{Calcium Silicate Coating Derived from Portland Cement}

A Portland cement-based is able to set in the presence of water and create a stable solid material. The small size (cumulative percentage of particle between 1.5-3 micron size may be approximately $70-80 \%$ ) makes the particles able to enter into open dentinal tubules. Portland cements mixed with water form a plastic and soft paste that may be applied and spread on dentine surfaces. The hydration of the Portland cement produces calcium hydroxide allowing the formation of apatite crystallites in the presence of phosphatecontaining biological fluids. According to Gandolfi et $\mathrm{al}^{44}$ (2008), the calcium silicate crystals have ability to penetrate inside dentinal tubules and resist to saliva immersion and to acid challenge, suggesting that this material obliterates tubules, protecting dentinal surface and may be used for dentine hypersensitivity therapy.

\section{Home-made Desensitizing Agents for Self-applied}

\section{Dentifrices}

Long used to treat dentinal hypersensitivity, dentifrices are cost-effective, noninvasive, simple to use and can be applied at home. ${ }^{16}$ Dentifrices with formulations containing potassium salts (e.g. chloride, nitrate, citrate, oxalate) and the others with fluoride are the most used home-made agents. $^{45}$

Formulations containing potassium salts perform a possible neural action mechanism by the diffusion of $\mathrm{K}^{+}$ through the dentinal tubules, increasing the extracellular potassium ion concentration, depolarizing the nerve fibers membranes and blocking the stimuli passage. Sensodyne total care toothpaste (Block Drug Corp, USA) is a dentifrice example that associates $6 \%$ potassium nitrate with sodium fluoride. Colgate sensitive fresh stripe toothpaste (ColgatePalmolive Ltd. USA) is a dentifrice example that associates $5.53 \%$ potassium citrate with sodium monofluorophosphate. Sensodyne total care F and Sensodyne total care gel (Block Drug Corp, USA) are dentifrice examples that use potassium chloride. It is important to highlight that no treatment, which the action mechanism is occlusive, must be done previously in the dental office because this will block the $\mathrm{K}^{+}$diffusion through dentinal tubules. ${ }^{46}$

Low-level fluoride dentifrices are not efficacious agents when used alone in the management of dentinal hypersensitivity. ${ }^{47}$

Hughes et $\mathrm{al}^{48}$ (2002) reported that amphiphilic organic materials have been used as an antisensitive teeth agent in the manufacture of toothpaste for sensitive teeth. Typical examples of such amphiphilic materials are fatty acid glycerides like glyceryl monooleate, glyceryl monolinoleate and glyceryl monoisostearate. Amphiphilic organic materials which are capable of forming, upon contact with moisture, a water-insoluble liquid crystal phase of at least one periodicity, have been found to have dentinal hypersensitivity reducing properties. ${ }^{48}$ 
Arginine is an amino acid naturally found in saliva composition. Recent research suggests that the arginine and calcium carbonate present in the formula of a toothpaste provides significantly increased dentin hypersensitivity relief. ${ }^{49-51}$ Arginine has the characteristic of presenting its molecule, at physiological $\mathrm{pH}$, positively charged. The desensitizing mechanism of action is due its molecule connect to the dentin surface, negatively charged, occluding the dentinal tubules. Colgate Sensitive Pro-Relief (ColgatePalmolive Ltd, USA) is a toothpaste example that associates $8 \%$ arginine and calcium carbonate.

\section{Mouthwashes}

Mouthwashes are utilized by hospitals, dentists and in general by consumers. Some commercially available mouthwashes contain a high percentage of ethyl alcohol. This formulations have some drawbacks: Alcoholcontaining mouthwashes contribute to the increased risk of development of oral cancer, ${ }^{52}$ a large number of persons cannot tolerate alcohol, alcohol-containing mouthwashes can be a poison hazard to a small child, alcohol-containing mouthwashes are often abused by alcoholics, alcohol in a mouthwash may irritate the protective layers of the mouth and throat, or dry out inflamed tissues. ${ }^{53}$ Listerine mouthwash buccal original (Johnson \& Johnson Ind. Com) is a mouthwash example that use alcohol at concentration of $26.9 \%$. Alcohol-free mouthwash can be fluoride, nonfluoride or association of two desensitizing agents.

Nonfluoride desensitizing mouthwash includes strontium salts (i.e. chloride, bromide, iodide, acetate, edetate, nitrate, salicylate and lactate) and potassium salts (i.e. chloride, bromide, iodide, acetate, citrate, nitrate and lactate). ${ }^{54}$ Thus, also include sodium chloride, sodium bicarbonate and flavoring agent. Sodium chloride and sodium bicarbonate produce a solution that is isotonic and thereby nonirritating to the oral mucosa, act to maintain and restore the integrity of the tissues of the mouth and throat. ${ }^{53}$

The fluoride salt most utilized in the mouthwash formulations is sodium fluoride. Fluoride desensitizing mouthwash formulations include sodium fluoride at concentration of the $0.2 \%$ are effective as desensitizing agent. ${ }^{55}$ The combination of the potassium nitrate at concentration of $3 \%$ and sodium fluoride at concentration of $0.2 \%$ have therapeutic potential to alleviate dentinal hypersensitivity. ${ }^{55,56}$

\section{Placebos}

It is commonly observed a dentinal hypersensitivity reduction of about 20 to $60 \%$ with the use of placebos, mainly as dentifrices. It occurs because of a complex association of physiologic and psychological interactions which depend considerably on the professional/patient relationship, with both parties believing in the treatment and desiring to obtain the relief of symptoms. In addition, the positive emotional and motivational behavioral responses can activate the body's central pain inhibiting system, which can modulate the peripheral painful stimuli through the release of endorphin centrally. ${ }^{57}$

\section{CONCLUSION}

Dentinal hypersensitivity involves a complex association of etiological factors. The patients should be advised about the preventive aspects, such as dietary acids, brushing techniques and balanced occlusion. The treatment is difficult and varied. Self-applied treatments, such as desensitizing dentifrices, are still large used as home-made desensitizing agent. The adhesive systems are one of the most effective clinical treatments and the lasers are expected to play an important role in treating dentinal hypersensitivity.

\section{REFERENCES}

1. Bissada NF. Symptomatology and clinical features of hypersensitive teeth. Arch Oral Biol 1994;39:Suppl.31-32S.

2. Flynn J, Galloway R, Orchandson R. The incidency of hypersentive teeth in the West Scotland. J Dent 1995;13: 230-36.

3. Berman LH. Dentinal sensation and hypersensitivity: Review of mechanisms and treatment alternatives. J Period 1985;56: 216-22.

4. Graf H, Galasse R. Morbidity, prevalence and intraoral distribution of hypersensitive teeth. J Dent Res 1977;56:Sp. Issue A162,230-36.

5. Lilja J. Innervation of different parts of the predentin and dentin in young human premolars. Acta Odontol Scand 1979;37: 339-46.

6. Dowell P, Addy M. Dentine hypersensitivity - a review. Aetiology, symptoms and theories of pain production. J Clin Periodont 1983;10:341-50.

7. Trowbridge HO. Review of dental pain: Histology and physiology. J Endod 1986;12:445-52.

8. Brännström M, Äström A. A study on the mechanisms of pain elicited from dentin. J Dent Res 1964;43:619-25.

9. Fogel HM, Marshall FJ, PAshley DH. Effects of distance from the pulp and thickness on the hidraulic condutance of human radicular dentin. J Dent Res 1998;67:1381-85.

10. Pashley DH. Dentine permeability, dentine sensitivity and treatment through tubule occlusion. J Endod 1986;12:465-74.

11. Garberoglio R, Brännström M. A scanning electron microscopic investigation of human dentinal tubules. Arch Oral Biol 1976;24:355-62.

12. Absi EG, Addy M, Adams D. Dentine hypersensitivity—a study of the patency of dentinal tubules in sensitive and nonsensitive cervical dentine. J Clin Periodontol 1987;14:280-84.

13. Ciucchi B, Bouillaguet S, Holz J, Pashley DH. In vivo estimation of pulpal pressure in humans. [Abstract 1359] J Dent Res 1993; 72: Issue 273. 
14. Kleinberg I, Kaufman HW, Wolff M. Measurement of tooth hypersensitivity and oral factors involved in its development. Arch Oral Biol 1994;39:Suppl. 63-71S.

15. Addy M, Pearce N. Aetiological, predisposing and environmental factors in dentine hypersensitivity. Arch Oral Biol 1994;39:Suppl. 33S-38S.

16. Wichgers TG, Emert RL. Dentine hypersensitivity. Gen Dent 1996;44:225-30.

17. McGrath PA. Psychological aspects of pain perception. Arch Oral Biol 1994;39:55-62S.

18. Olgart L, Kerezoudis NP. Nerve-pulp interactions. Arch Oral Biol 1994;39:47-54S.

19. Addy M, West N. Etiology, mechanisms and management of dentine hypersensitivity. Current Opinion Periodontics 1994; 71-77.

20. Grossman L. The treatment of hypersensitive dentin. J Am Dent Assoc 1935:22:592-602.

21. Zappa U. Self-applied treatments in the management of dentine hypersensitivity. Arch Oral Biol 1994;39:107-112S.

22. Bal J, Kundalgurki S. Tooth sensitivity prevention and treatment. Oral Health 1999;89(2):33-34;37-38,41.

23. Gangarosa LP. Current strategies for dentist-applied tratment in the management of hypersensitive dentine. Arch Oral Biol 1994;39:101-06S.

24. Olusile AO, Bamise CT, Oginni AO, Dosumu OO. Short-term clinical evaluation of four desensitizing agents. J Contemp Dent Pract 2008;9(1):22-29.

25. Pereira JC, Segala AD, Gillam DG. Effect of desensitizing agents on the hydraulic conductance of human dentin subjected to different surface pretreatments - an in vitro study. Dent Mater 2005;21(2):129-38.

26. Dood GP, Markowitz KJ. Dentin desensitizer containing stannous fluoride. United States Patent EP 6592853;2003B.

27. Charvat J, Soremark R, Li J, Vacek J. Titanium tetrafluoride for treatment of hypersensitive dentine. Swed Dent J 1995;19: 41-46.

28. Kazemi RB, Sen BH, Spangberg LSW. Permeability changes of dentine treated with titanium tetrafluoride. J Dent 1999;27: $531-38$

29. Singal P, Gupta R, Pandit N. Two percent sodium fluorideiontophoresis compared to a commercially available desensitizing agent. J Periodontol 2005;76(3):351-57.

30. Ross MR. Hypersensitive teeth. Effect of strontium chloride in a compatible dentifrice. J Periodontol 1961;32:49.

31. Kishore A, Mehrotra KK, Saimbi CS. Effectiveness of desensitizing agents. J Endod 2002;28(1):34-35.

32. Madhu PS, Setty S, Ravindra S. Dentinal hypersensitivity?Can this agent be the solution? Indian J Dent Res 2006; 17(4):178-84.

33. Dondi dall'Orologio G, Lorenzi R, Anselmi M, Opisso V. Dentin desensitizing effects of Gluma Alternate, Health-Dent Desensitizer and Scotchbond Multi-Purpose. Am J Dent 1999;12(3):103-06.

34. Türkün SL. Clinical evaluation of a self-etching and a one-bottle adhesive system at two years. J Dent 2003;31(8):527-34.

35. Liu HC, Lin CP, Lan WH. Sealing depth of Nd:YAG laser on human dentinal tubules. J Endod 1997;23:691-93.

36. Hsu PJ, Chen JH, Chuang FH, Roan RT. The combined occluding effects of fluoride-containing dentin desensitizer and Nd-Yag laser irradiation on human dentinal tubules: An in vitro study. Kaohsiung J Med Sci 2006;22(1):24-29.
37. Kumar NG, Mehta DS. Short-term assessment of the Nd:YAG laser with and without sodium fluoride varnish in the treatment of dentin hypersensitivity - a clinical and scanning electron microscopy study. J Periodontol 2005;76(7):1140-47.

38. Birang R, Poursamimi J, Gutknecht N, Lampert F, Mir M. Comparative evaluation of the effects of Nd:YAG and Er:YAG laser in dentin hypersensitivity treatment. Lasers Med Sci 2007;22(1):21-24.

39. Schwarz F, Arweiler N, Georg T, Reich E. Desensitizing effects of an Er:YAG laser on hypersensitive dentine. J Clin Periodontol 2002;29(3):211-15.

40. Zhang $\mathrm{C}$, Matsumoto $\mathrm{K}$, Kimura $\mathrm{Y}$, et al. Effects of $\mathrm{CO}_{2}$ laser in treatment of cervical dentinal hypersensitivity. J Endod 1998;24(9):595-97.

41. Slutzky-Goldberg I, Nuni E, Nasralla W, Stabholtz A, Moshonov J. The effect of $\mathrm{CO}_{2}$ laser on the permeability of dentinal tubules: A preliminary in vitro study. Photomed Laser Surg 2008;26(1):61-64.

42. Walsh LJ. The current status of low level laser therapy in dentistry. Part 2. Hard tissue applications. Aust Dent J 1997;42:302-06.

43. Tengrungsun T, Sangkla W. Comparative study in desensitizing efficacy using the GaAlAs laser and dentin bonding agent. J Dent 2008;36(6):392-95.

44. Gandolfi MG, Silvia F, H PD, Gasparotto G, Carlo P. Calcium silicate coating derived from Portland cement as treatment for hypersensitive dentine. J Dent 2008;36(8):565-78.

45. Orchardson R, Gillam DG. The efficacy of potassium salts as agents for treating dentin hypersensitivity. J Orofac Pain 2000;14(1):9-19.

46. Hodosh M, Hodosh SH, Hodosh AJ. About dentinal hypersensitivity. Compend Contin Educ Dent 1994;15:658-67.

47. Plagmann HC, König J, Bernimoulin JP, Rudhart AC, Deschner J. A clinical study comparing two high-fluride dentifrices for the treatment of dentinal hypersensitivity. Quintessence Int 1997;28:403-08.

48. Hughes NP, Littlewood DL, Stead WJ. Desensitising toothpaste. European Patent EP0868167;2002.

49. Docimo R, Montesani L, Maturo P, Costacurta M, Bartolino $\mathrm{M}$, DeVizio $\mathrm{W}$, et al. Comparing the efficacy in reducing dentin hypersensitivity of a new toothpaste containing $8.0 \%$ arginine, calcium carbonate, and $1450 \mathrm{ppm}$ fluoride to a commercial sensitive toothpaste containing $2 \%$ potassium ion: An eightweek clinical study in Rome, Italy. J Clin Dent 2009;20(1): 17-22.

50. Docimo R, Montesani L, Maturo P, Costacurta M, Bartolino $\mathrm{M}$, Zhang YP, et al. Comparing the efficacy in reducing dentin hypersensitivity of a new toothpaste containing $8.0 \%$ arginine, calcium carbonate and $1450 \mathrm{ppm}$ fluoride to a benchmark commercial desensitizing toothpaste containing $2 \%$ potassium ion: an eight-week clinical study in Rome, Italy. J Clin Dent 2009;20(4):137-43.

51. Ayad F, Ayad N, Zhang YP, DeVizio W, Cummins D, Mateo LR. Comparing the efficacy in reducing dentin hypersensitivity of a new toothpaste containing $8.0 \%$ arginine, calcium carbonate, and $1450 \mathrm{ppm}$ fluoride to a commercial sensitive toothpaste containing 2\% potassium ion: An eight-week clinical study on Canadian adults. J Clin Dent 2009;20(1):10-06.

52. McCullough MJ, Farah CS. The role of alcohol in oral carcinogenesis with particular reference to alcohol-containing mouthwashes. Aust Dent J 2008;53(4):302-05. 
53. Thompson WJ. Mouthwash. United States Patent 1992; 514:5664.

54. Jackson RJ, Duke SA, Wicks MA. Nonfluoride desensitizing mouthwash. United States Patent 1992;5087444.

55. Pereira R, Chava VK. Efficacy of a 3\% potassium nitrate desensitizing mouthwash in the treatment of dentinal hypersensitivity. J Periodontol 2001;72(12):1720-25.

56. Gillam DG, Bulman JS, Jackson RJ, Newman HN. Efficacy of a potassium nitrate mouthwash in alleviating cervical dentine sensitivity (CDS). J Clin Periodontol 1996;23(11):993-97.

57. Trowbridge HO, Silver DR. A review of current approaches to in-office management of tooth hypersensitivity. Dent Clin North Am 1990;34(3):561-81.

\section{ABOUT THE AUTHORS}

\section{Alessandra Bühler Borges}

Assistant Professor, Department of Restorative Dentistry, São José dos Campos School of Dentistry, São Paulo State University São Paulo, Brazil

\section{Daphne Câmara Barcellos}

Postgraduate Student, Department of Restorative Dentistry, São José dos Campos School of Dentistry, São Paulo State University, São Paulo Brazil

\section{Carlos Rocha Gomes Torres}

Assistant Professor, Department of Restorative Dentistry, São José dos Campos School of Dentistry, São Paulo State University São Paulo, Brazil

\section{Alexandre Luiz Souto Borges}

Assistant Professor, Department of Dental Materials and Prosthodontics, São José dos Campos School of Dentistry, São Paulo State University, São Paulo, Brazil

\section{Ana Lucia Marsilio}

Clinical Research Academic Group, São José dos Campos School of Dentistry, São Paulo State University, São Paulo, Brazil

\section{Cláudio Antonio Talge Carvalho}

Assistant Professor, Department of Restorative Dentistry, Endodontics Course, São José dos Campos School of Dentistry, São Paulo State University, São Paulo, Brazil

\section{CORRESPONDING AUTHOR}

Carlos Rocha Gomes Torres, Assistant Professor, Avenida Engenheiro Francisco José Longo, 777, Jardim São Dimas São José dos Campos, CEP: 12245-000, São Paulo, Brazil, Phone: (12) 3947 9048, Fax: (12) 3947 9010, e-mail: carlosrgt@fosjc.unesp.br 